\section{PREFERENTIAL INCORPORATION OF TUNICAMYGIN, AN \\ ANTIVIRAL ANTIBIOTIC CONTAINING GLUCOSAMINE, INTO THE CELL MEMBRANES}

\section{Sir :}

Tunicamycin (tunica means coat) is an antiviral, glucosamine-containing antibiotic ${ }^{1}$ which inhibits the incorporation of sugars into cell membranes and acid-insoluble products ${ }^{2}$. Some aminosugar derivatives were found to reverse the antiviral activity of tunicamycin against Newcastle disease virus $(\mathrm{NDV})^{3)}$. The biosynthesis of neuraminidase and hemagglutinin, glycoproteins, was preferentially inhibited by the antibiotic at low drug dosages in comparison with that of other virus components such as capsid, RNA-dependent RNA polymerase and NDVRNA (unpublished observations). Such observations suggest that tunicamycin is incorporated into cellular or viral macromolecular fractions as an analogue of aminosugars or sugar-containing precursors. We have investigated the incorporation of tunicamycin into cellular components using isotopically labeled antibiotic.

Tritiated tunicamycin was obtained from Dai-Ichi Chemicals, Tokyo. Monodispersed cells were prepared by treatment of 9 - to 11-day-old chick embryos with $0.25 \mathrm{w} / \mathrm{v} \%$ trypsin.

Monodispersed cells in supplemented GEY's salt solution were incubated in the presence of ${ }^{3} \mathrm{H}$-tunicamycin at $37^{\circ} \mathrm{C}$. Sample portions $(1.0 \mathrm{ml})$ were withdrawn at regular time intervals. Cells were collected by centrifugation, washed, and broken by freezingand-thawing. Membrane fractions were collected by centrifugation and washed. The supernatants and the washes were combined, made $5 \%$ with trichloroacetic acid (TCA), and the precipitates were collected on membrane filters (cytoplasmic fraction). Radioactivity in both fractions was counted. Time course of the incorporation of ${ }^{3} \mathrm{H}$ tunicamycin into the membrane and the cytoplasmic fractions is shown in Fig. 1. Radioactivity in both fractions increased linearly for the first 8 hours at least. Tunicamycin was incorporated preferentially into the membrane fraction, and the radioactivity in the membrane fraction was 15 times more than that in the cytoplasmic fraction.

The result presented in Fig. 1 suggests specific incorporation of tunicamycin into the cell membranes. To investigate whether the antibiotic found in the membrane fraction is simply bound to or metabolically incorporated into the cell membranes, cells were treated with $0.1 \%$ trypsin or $10^{-2} \mathrm{M}$ ethylenediaminetetraacetic acid (EDTA) at $37^{\circ} \mathrm{C}$ for 10 minutes after the incubation with ${ }^{3} \mathrm{H}$-tunicamycin. Such treatment released little ${ }^{3} \mathrm{H}$-tunicamycin from the cell membranes (Fig. 2). The treatment with trypsin after 1 -hour period of incubation solubilized about $50 \%$ of the radioactivity in the membrane fraction, but the ratio of

Fig. 1. Incorporation of ${ }^{3} \mathrm{H}$-tunicamycin into the membrane and cytoplasmic fractions Twenty milliliters of monodispersed chick embryo fibroblasts (ca. $5 \times 10^{6}$ cells $/ \mathrm{ml}$ ) in the GEY's salt solution supplemented with lactalbumin hydrolysate, yeast extract and calf serum ${ }^{2}$ were incubated at $37 \mathrm{C}$ with occasional stirring in the presence of $5 \mathrm{~kg} / \mathrm{m} 1{ }^{3} \mathrm{H}$-tunicamycin. Sample portions $(2.0$ mi) were withdrawn at regular time intervals and chilled in an ice-water bath. Cells were collected by centrifugation $(1,500 \times g .15$ minutes), washed thoroughly with phosphate-buffered $(0.05 \mathrm{M}, \mathrm{pH} 7.3)$ physiological saline, and broken by freezing-andthawing five times in a dry ice-acetone bath. Membrane fractions were collected by centrifugation and washed with $1.0 \mathrm{ml}$ cold buffered saline two times. The supernatants and the washes of the broken cells were combined, made $5 \%$ with cold TCA, and precipitates (cytoplasmic fraction) were collected on membrane filters (pore size $0.45 \mathrm{~m} \mu$; Millipore Corp.). Radioactivity retained on the filters was counted in $10 \mathrm{~m} 1$ scintillation liquid ${ }^{2)}$, and the radioactivity at 0 hour was subtracted from that of each sample.

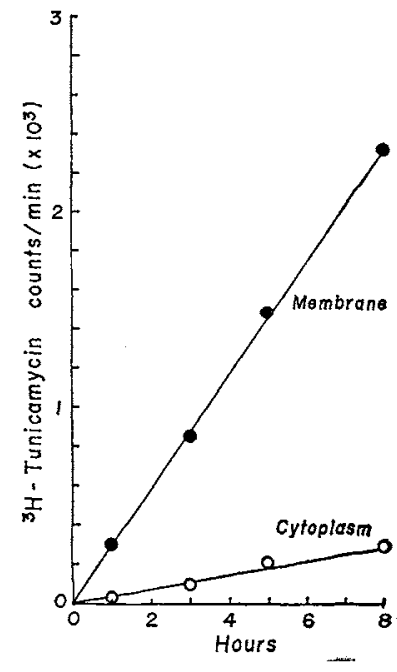


Fig. 2. Effect of treatment with trypsin or EDTA on ${ }^{3} \mathrm{H}$-tunicamycin incorporated into the cell membranes

Fourty milliliters of cell suspension in the supplemented GEY's salt solution (ca. $5 \times 10^{6} \mathrm{cells} / \mathrm{mi}$ ) were incubated in the presence of $5 \mu \mathrm{g} / \mathrm{ml}^{3} \mathrm{H}$-tunicamycin at $37^{\circ} \mathrm{C}$ with occasional stirring. At designated time intervals, $5.0 \mathrm{ml}$ sample portions were withdrawn and chilled in melting ice. Cells were collected and washed as described in the legend to Fig. 1 . The washed cells were resuspended in $3.0 \mathrm{ml}$ of the buffered saline and divided evenly into three portions. Two were treated with $0.1 \%$ trypsin or $10^{-2}$ $M$ EDTA at $37^{\circ} \mathrm{C}$ for 10 minutes, and the other served as control. The treated cells were collected by centrifugation, washed thoroughly, and suspended in $2.0 \mathrm{ml}$ of the buffered saline. The cells were broken by freezing-and-thawing and filtered through membrane filters. The cell membranes retained on the filters were washed with cold $0.1 \mathrm{~N}$ $\mathrm{KOH}$ containing $0.8 \% \mathrm{NaCl}$. Radioactivity in the mebrane fractions was counted.

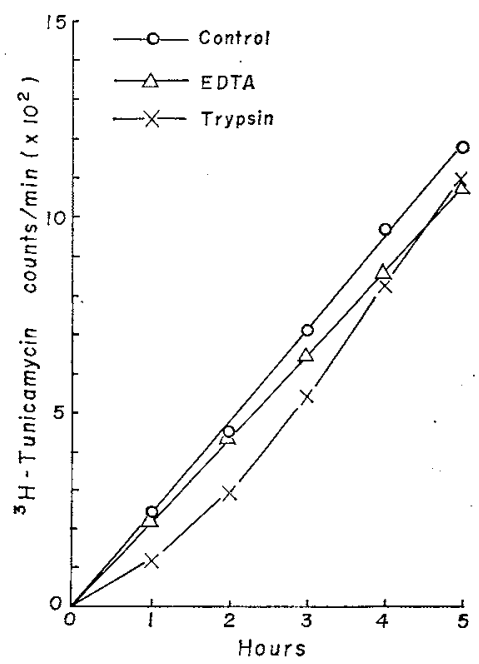

the solubilized radioactivity decreased as the time of incubation increased.

The antiviral activity of tunicamycin was found to be partially reversed by some aminosugar derivatives ${ }^{3)}$. If the antiviral activity is attributable to altered synthesis of the cell membranes caused by the incorporation of tunicamycin into the cell membranes, aminosugar derivatives which effectively reverse the antiviral activity of tunicamycin may interfere with the incorporation of the antibiotic into the cell membranes. As shown in Fig. 3, N-acetylglucosamine, one of the effective aminosugars, decreased the incorporation of ${ }^{3} \mathrm{H}-$ tunicamycin into the membrane fraction.

The result of the treatment with trypsin or EDTA oppose the possibility of simple binding of tunicamycin to the cell membranes as observed with macromomycin,
Fig. 3. Effect of N-acetyl-D-glucosamine on the incorporation of ${ }^{3} \mathrm{H}$-tunicamycin into the cell membranes

Sixty milliliters of cell suspension $\left(c a .5 \times 10^{6} \mathrm{cells} /\right.$ mil) were divided into equal four portions. Specified concentrations of N-acetyl-D-glucosamine were added to three, and the other served as control. Two milliliters of sample portions were withdrawn at designated time intervals and radioactivity in the membrane fractions was counted as described in the legend to Fig. 2.

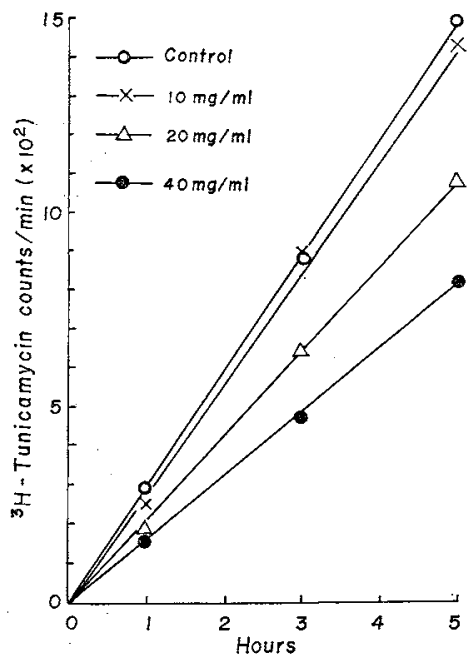

which is reversibly bound to the cell membranes $^{4)}$. The observations that tunicamycin was preferentially incorporated into the cell membranes and $\mathrm{N}$-acetylglucosamine interfered with the incorporation are consistent with the assumption that tunicamycin is incorporated into the cell membranes instead of sugars or precursors of sugar-containing cellular components and alters membrane synthesis. Altered membranes synthesized in the presence of tunicamycin may be the cause of the antiviral activity of the antibiotic and the different behavior of the cells grown in the presence of the antibiotic to phytohemagglutinin. Morphological changes of microbial cells induced by tunicamycin ${ }^{\text {s) }}$ may also be caused by a similar action mechanism of the antibiotic. The cell envelope of mammalian and microbial cells has attracted interest lately and evidence is accumulating that the cell envelope plays an important role in virus multiplication ${ }^{6,7,8)}$ and cell division including malignant transformation ${ }^{9,10,11,12)}$. Tunicamycin is one of the rare inhibitors of membrane synthesis and might become a useful tool for study of the synthesis and function of mammalian and microbial cell envelope. 
The details of this study will be reported elsewhere.

\section{Acknowledgement}

This investigation was financially supported by grant-in-aid from Waksman Foundation of Japan.

\section{Akira Takatsuki \\ Gakuzo Tamura}

Laboratory of Microbiology

Department of Agricultural Chemistry

The University of Tokyo

Bunkyo-ku, Tokyo, Japan

(Received December 15, 1971)

\section{References}

1) Takatsukr, A.; K. Arima \& G. Tamura : Tunicamycin, a new antibiotic. I. Isolation and characterization of tunicamycin. J. Antibiotics $24: 215 \sim 223,1971$

2) Takatsuki, A. \& G. Tamura: Effect of tunicamycin on the synthesis of macromolecules in cultures of chick embryo fibroblasts infected with Newcastle disease virus. J. Antibiotics $24: 785 \sim 794,1971$

3) Takatsukr, A. \& G. Tamura : Tunicamycin, a new antibiotic. III. Reversal of the antiviral activity of tunicamycin by aminosugars and their derivatives. J. Antibiotics $24: 232 \sim 238,1971$

4) Kunmoto, T.; M. Hori \& H. Umezawa : Reversible binding of macromomycin, a macromolecular peptide antibiotic, to cell membranes. I. Antibiotics $24: 203 \sim 205,1971$

5) Takatsuki, A.; K. Shimizu \& G. Tamura: Effect of tunicamycin on microorganisms: Morphological changes and degradation of RNA and DNA induced by tunicamycin. J. Antibiotics $25: 75 \sim 85,1972$

6) Keller, J. M.; P. G. Spear \& B. Roizman : Proteins specified by herpes simplex virus. III. Viruses differing in their effect on the social behavior of infected cells specify different membrane glycoproteins. Proc. Nat. Acad. Sci. $65: 865 \sim 871,1970$

7) Burge, B.W. \& J.H. Strauss : Glycopeptides of the membrane glycoprotein of Sindbis virus. J. Mol. Biol. $47: 449 \sim 466,1970$

8) Holland, J. J. \& E. D. KrehN : Influenza virus effects on cell membrane proteins. Science $167: 202 \sim 205,1970$

9) Rogers, H. J.: Bacterial growth and the cell envelope. Bacteriol. Rev. $34: 194 \sim 214$, 1970

10) Stoker, M.; C. O'Neill, S. Berryman \& V. WAKSMAN : Anchorage and growth regulation in normal and virus-transformed cells. Int. J. Cancer $3: 683 \sim 693,1968$

11) Burger, M. M. \& A.R. GoldberG: Identification of a tumor-specific determinant on neoplastic cell surfaces. Proc. Nat. Acad. Sci. $57: 359 \sim 366,1967$

12) Merzan, E.; H.C. Wu, P.H. Bluack \& P.W. Robrins : Comparative studies on the carbohydrate-containing membrane components of normal and virus-transformed mouse fibroblasts. II. Separation of glycoproteins and glycopeptides by Sephadex chromatography. Biochemistry $8: 2518 \sim 2524,1969$ 